\title{
Colitis isquémica secundaria a infección por citomegalovirus en un paciente con enfermedad renal crónica
}

\section{Ischemic colitis, secondary to CMV infection in a patient with chronic kidney disease}

\author{
Héctor M. Delgado-Cortés ${ }^{1 *}$, Susanna I. Scharrer-Cabello ${ }^{1}$, Héctor J. Maldonado-Garza ${ }^{1}$ \\ y EdWARS A. RodríGuez-HINOJOSA ${ }^{2}$
}

${ }^{1}$ Departamento de Gastroenterología, ²Departamento de Patología. Hospital Universitario Dr. José Eleuterio González, Monterrey, N.L, México

\begin{abstract}
We present the clinical case of a 72-year-old male with a history of diabetes mellitus, systemic arterial hypertension and chronic kidney disease who, days after starting hemodialysis therapy, presented a clinical picture of hematochezia. A diagnostic colonoscopy was performed, finding ischemic pancolitis. The histopathological report corroborated this and the subsequent immunohistochemistry showed the presence of cytomegalovirus (CMV). Ischemic colitis in the context of CMV infection is one of the less common forms, so the diagnosis requires high clinical suspicion and the use of targeted paraclinical studies.
\end{abstract}

Key words: Ischemic colitis. Cytomegalovirus. Chronic kidney disease. Hemodialysis.

\section{RESUMEN}

Se presenta el caso clínico de un hombre de 72 años de edad con antecedentes de diabetes mellitus, hipertensión arterial sistémica y enfermedad renal crónica, que días después de iniciar terapia con hemodiálisis presentó cuadro clínico de hematoquecia. Se realizó colonoscopia diagnóstica, encontrando pancolitis isquémica. El reporte histopatológico lo corroboró y la posterior inmunohistoquímica evidenció la presencia de citomegalovirus (CMV). La colitis isquémica en el contexto de infección por CMV es una de las formas menos comunes, por lo que el diagnóstico requiere de una alta sospecha clínica y el uso de estudios paraclínicos en forma dirigida.

Palabras clave: Colitis isquémica. Citomegalovirus. Enfermedad renal crónica. Hemodiálisis.

\section{Correspondence:}

*Héctor M. Delgado-Cortes

E-mail: hectorm.delgado@gmail.com
Date of reception: 22-04-2020

Date of acceptance: 15-04-2021

DOI: 10.24875/HMCM.20000025
Available online: $25-06-2021$

Hosp Med Clin Manag. 2020;13:164-8

2604-0018 / @ 2021 Mexican Regional Hospitals of High Specialty and Federal Hospitals. Published by Permanyer. This is an open access article under the CC BY-NC-ND license (http://creativecommons.org/licenses/by-nc-nd/4.0/). 


\section{INTRODUCCIÓN}

La colitis por citomegalovirus (CMV) ha sido típicamente descrita en pacientes con enfermedad inflamatoria intestinal o en estado de inmunosupresión. De forma clásica este grupo de pacientes incluye a aquellos con infección por el virus de la inmunodeficiencia humana $(\mathrm{VIH})$ en estadios avanzados, receptores de trasplante, pacientes sometidos a quimioterapia antineoplásica por cáncer y en aquellos pacientes con uso crónico de esteroides $^{1,2}$. La infección sintomática por CMV en el paciente no inmunocomprometido por lo regular es de evolución benigna y autolimitada, caracterizada por un síndrome mononucleósico típico; sin embargo, cada vez hay más reportes de casos con manifestaciones clínicas severas ${ }^{3}$.

A nivel gastrointestinal, el CMV puede afectar desde el esófago hasta el colon ${ }^{4}$; sin embargo, la colitis por CMV por lo regular presenta afección segmentaria y los hallazgos en la colonoscopia, aunque pueden ser diversos, corresponden a lesiones inflamatorias, úlceras de base ancha y lesiones exofíticas irregulares, en algunos casos llegando a simular lesiones tumorales ${ }^{1,5,6}$.

Presentamos el caso de un paciente con inmunidad aparentemente conservada que desarrolló pancolitis isquémica, una de las formas menos frecuentes de presentación.

\section{CASO CLÍNICO}

Paciente de sexo masculino de 72 años de edad, con antecedente de diabetes mellitus tipo 2 diagnosticada hace 30 años, con mal apego a tratamiento. Hipertensión arterial sistémica diagnosticada hace 30 años y en tratamiento farmacológico con amlodipino $5 \mathrm{mg}$ vía oral cada 12 horas. Enfermedad renal crónica diagnosticada en enero de 2019 sin recibir tratamiento específico previo a su padecimiento actual. Acude al servicio de urgencias por hipoglucemia sintomática además de sobrecarga hídrica. Se inicia terapia sustitutiva de la función renal con hemodiálisis, teniendo el paciente una evolución clínica adecuada en los días subsecuentes. Sin embargo, posterior a la 4. ${ }^{a}$ sesión de hemodiálisis presenta hematoquecia franca en seis ocasiones, sin otra sintomatología. El examen físico no reveló datos adicionales; a nivel abdominal no se encontraron regiones dolorosas o presencia de lesiones palpables y el

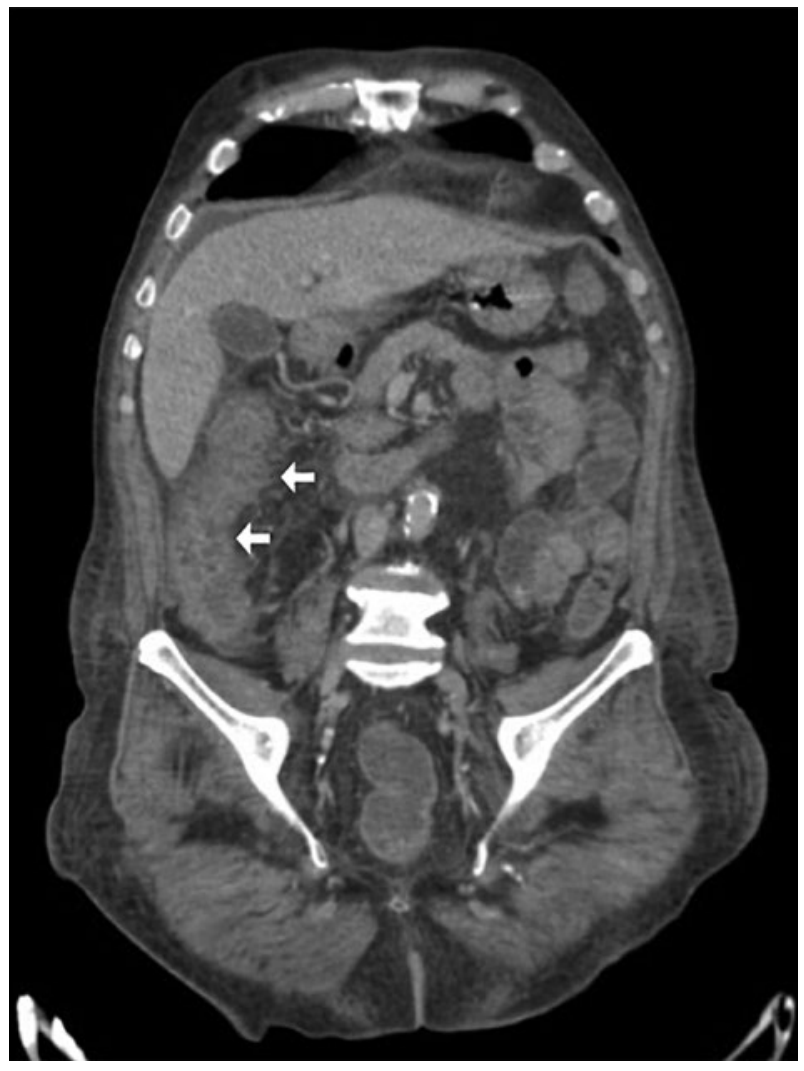

Figura 1. Tomografía coronal simple de abdomen. Las flechas hacia colon ascendente muestran engrosamiento de su pared.

tacto rectal fue positivo para hematoquecia sin encontrar otras alteraciones. Exámenes paraclínicos revelaron una hemoglobina de $7.9 \mathrm{~g} / \mathrm{dl}$, leucocitos totales en $10.5 \mathrm{~K} / \mu \mathrm{l}$ con neutrofilia de $9.46 \mathrm{~K} / \mu \mathrm{l}$; además, alteraciones en la química sanguínea secundarios a la enfermedad renal con creatinina $11.1 \mathrm{mg} / \mathrm{dl}$ y nitrógeno ureico en sangre de 64. El panel viral para VIH fue negativo. Un estudio de tomografía simple realizado identificó engrosamiento de pared de colon transverso y ascendente con realce de esta y borramiento de planos grasos adyacentes sugestivo de colitis a ese nivel, sugiriendo una etiología infecciosa (Fig. 1). Se decide realizar colonoscopia, encontrando mucosa de ciego, colon ascendente, transverso y descendente de aspecto eritematoso con múltiples erosiones, mucosa friable, con patrón vascular perdido y haustral conservado, colon sigmoides y recto con erosiones eritematosas circulares hasta el margen anal. La impresión diagnóstica en este procedimiento fue de probable colitis isquémica (Fig. 2).

Se tomaron múltiples biopsias durante el procedimiento reportándose daño vascular, erosión del epitelio 


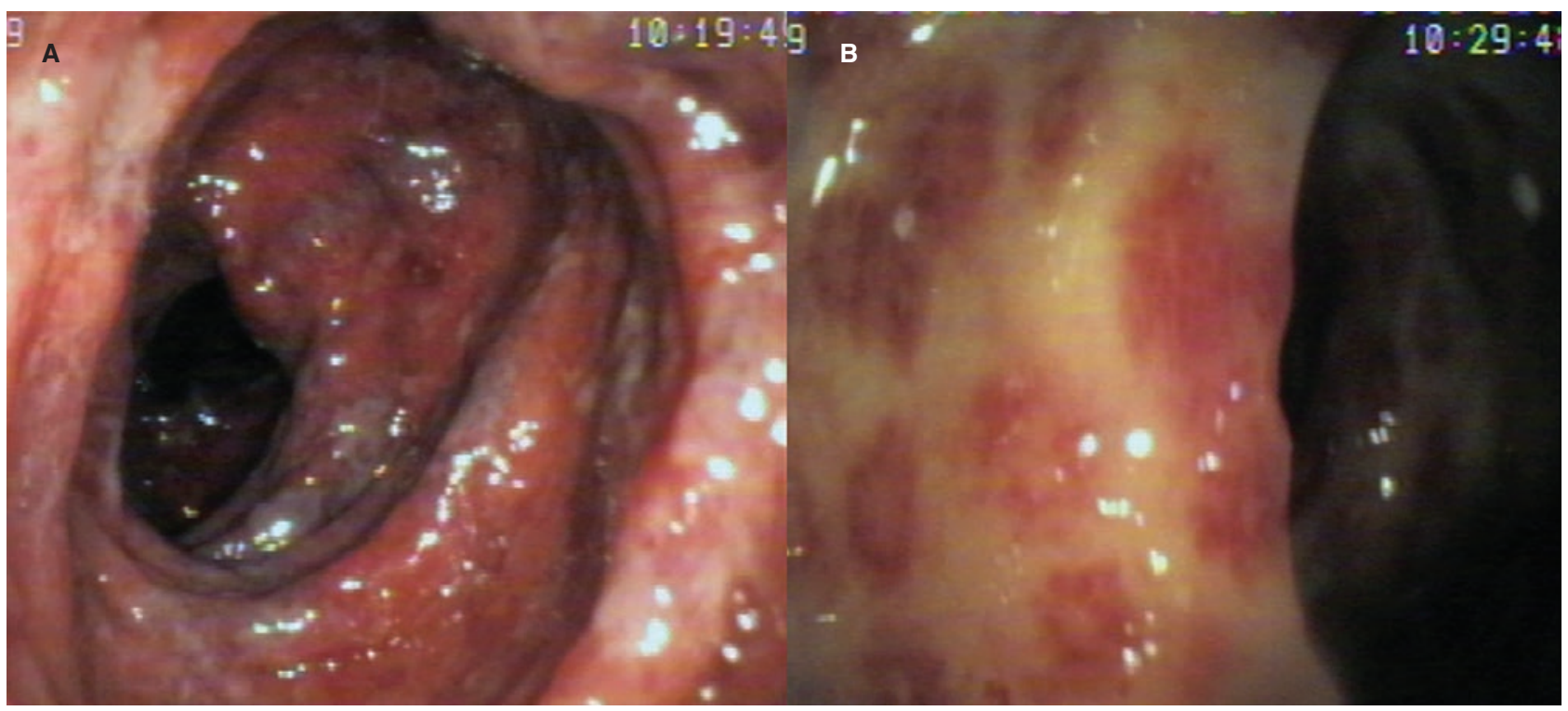

Figura 2. Hallazgos de la colonoscopia. Se observa mucosa eritematosa con pérdida del patrón vascular normal, datos sugestivos de colitis isquémica. A: mucosa de colon transverso. B: mucosa de recto.

superficial, extravasación de eritrocitos y polimorfonucleares con cambios reactivos de las glándulas colónicas consistentes con una colitis isquémica. Sin embargo, algunas células mostraban datos sugestivos de infección por CMV, motivo por el cual se realizaron tinciones de inmunohistoquímica, con resultado positivo para CMV (Fig. 3).

Se inició tratamiento con ganciclovir, a pesar de lo cual el paciente presentó evolución clínica tórpida, falleciendo al día 11 de tratamiento.

\section{DISCUSIÓN}

En la actualidad existen pocos reportes de casos de pacientes con colitis isquémica asociada a la infección por $\mathrm{CMV}^{4,7}$. Los factores de riesgo más comúnmente asociados son: edad avanzada, diabetes mellitus, hipertensión arterial, enfermedades cardiacas, enfermedad renal crónica, historial clínico de transfusiones sanguíneas y pacientes postrados en cama ${ }^{1,2,7}$. Varios de estos factores estaban presentes en nuestro paciente. Con respecto a las manifestaciones clínicas, las más frecuentemente descritas son: hematoquecia, diarrea, dolor abdominal, fiebre y la presencia de masa abdominal palpable ${ }^{2,7}$. En cuanto a los hallazgos de la colonoscopia, se han descrito patrones muy heterogéneos; predominan los cambios inflamatorios con pérdida o no del patrón vascular normal, úlceras múltiples o aisladas, exudados mucopurulentos y en algunos casos se ha descrito la formación de pseudomembranas (indistinguibles de la infección por Clostridioides difficile) ${ }^{1}$. Se han reportado lesiones exofíticas grandes que pueden semejar tumores ${ }^{6} \mathrm{y}$, pocas veces reportado, el patrón de colitis isquémica ${ }^{7}$. En cuanto a los exámenes paraclínicos, el rendimiento diagnóstico de la reacción en cadena de la polimerasa (PCR) para CMV, ya sea sérica o tisular, no ha sido adecuadamente determinado; sin embargo, se considera que la inmunohistoquímica para CMV es el método diagnóstico más aceptable ${ }^{8}$, el cual fue positivo en nuestro caso.

La relación entre la colitis isquémica y la infección por CMV no está completamente establecida. Se han propuesto mecanismos fisiopatológicos en los cuales el virus puede actuar tanto como un factor desencadenante, generando daño celular directo sobre el endotelio y la mucosa colónica, como también puede ser un oportunista que afecta una mucosa previamente dañada o con mecanismos de defensa deteriorada, esto último frecuentemente visto en pacientes inmunosuprimidos o con enfermedad inflamatoria intestinal9-12.

Los pacientes en hemodiálisis tienen mayor riesgo de exposición al CMV, ya sea por contacto con máquinas contaminadas o por la necesidad en algunos casos de terapia transfusional ${ }^{13,14}$. Además, se ha descrito el deterioro "leve» de la función inmunitaria asociada a la 


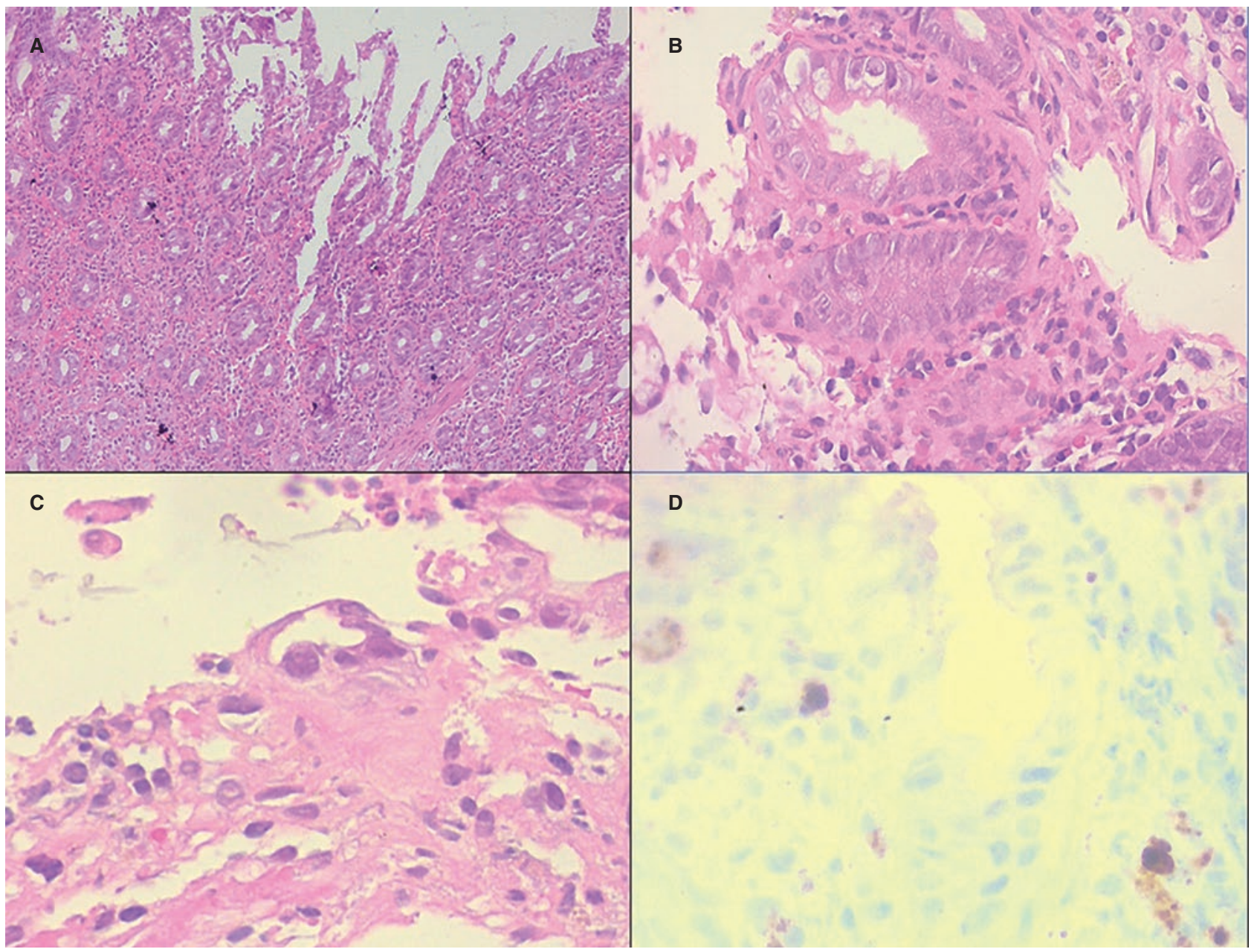

Figura 3. Mucosa de colon. A: tinción de hematoxilina y eosina (HyE) 40x. La imagen histológica sugiere daño vascular por isquemia; se observa erosión del epitelio superficial, con extravasación de eritrocitos y polimorfonucleares con cambios reactivos de las glándulas colónicas. B: HyE 100x: algunas células de las criptas y endotelios vasculares muestran agrandamiento nuclear e hipercromasia; algunas de las células muestran cambios sugestivos de infección por citomegalovirus (CMV). C: aumento 100x. D: la inmunohistoquímica muestra positividad nuclear con anticuerpo para CMV.

enfermedad renal crónica como causa suficiente para desarrollar colitis por CMV en este grupo de pacientes ${ }^{9}$. En el caso que presentamos, el desarrollo de esta entidad concuerda con el inicio reciente de la terapia sustitutiva, por lo cual proponemos que el CMV se comportó como causa desencadenante de la colitis isquémica (probablemente una reinfección), en lugar de como una infección oportunista.

Otros mecanismos de la colitis con patrón de isquemia que presentó el paciente pudieron ser la presencia de disfunción endotelial frecuentemente asociada a diabetes mellitus e hipertensión arterial, como factores predisponentes, siendo la infección por el CMV y los cambios vasculares funcionales asociados al inicio de la hemodiálisis los factores desencadenantes. Nuestro paciente no contaba con otra sintomatología asociada, como el estreñimiento ni consumo de fármacos asociados al desarrollo de colitis isquémica ${ }^{14}$.

El tratamiento antiviral con ganciclovir se encuentra indicado; aun así, la mortalidad asociada a la colitis por CMV es alta, reportándose cifras de hasta el $35 \%$ en algunos casos ${ }^{8}$.

\section{CONCLUSIÓN}

En los pacientes no inmunocomprometidos, el diagnóstico de colitis por CMV requiere de una gran sospecha clínica, por lo que en cuadros de colitis atípicos y en pacientes con múltiples comorbilidades se necesita el 
apoyo de técnicas diagnósticas adicionales específicas, destacando el uso de pruebas no invasivas de imagen, la colonoscopia con toma de biopsias y la histopatología.

\section{FINANCIAMIENTO}

Los autores no recibieron patrocinio para llevar a cabo este trabajo.

\section{CONFLICTO DE INTERESES}

Los autores declaran no tener conflicto de intereses alguno.

\section{RESPONSABILIDADES ÉTICAS}

Protección de personas y animales. Los autores declaran que para esta investigación no se han realizado experimentos en seres humanos ni en animales.

Confidencialidad de los datos. Los autores declaran que han seguido los protocolos de su centro de trabajo sobre la publicación de datos de pacientes.

Derecho a la privacidad y consentimiento informado. Los autores han obtenido el consentimiento informado de los pacientes y/o sujetos referidos en el artículo. Este documento obra en poder del autor de correspondencia.

\section{BIBLIOGRAFÍA}

1. Einbinder $Y$, Wolf DG, Pappo O, Migdal A, Tsvang E, Ackerman Z. The clinical spectrum of cytomegalovirus colitis in adults. Aliment Pharmacol Ther. 2008;27(7):578-87.

2. Ko JH, Peck KR, Lee WJ, Lee JY, Cho SY, Ha YE, et al. Clinical presentation and risk factors for cytomegalovirus colitis in immunocompetent adult patients. Clin Infect Dis. 2015;60(6):20-6.

3. Rafailidis PI, Mourtzoukou EG, Varbobitis IC, Falagas ME. Severe cytomegalovirus infection in apparently immunocompetent patients: a systematic review. Virol J. 2008;5:47.

4. Siegal DS, Hamid N, Cunha BA. Cytomegalovirus colitis mimicking ischemic colitis in an immunocompetent host. Heart Lung J Crit Care. 2005;34(4): 291-4.

5. Chen Y-M, Hung Y-P, Huang C-F, Lee N-Y, Chen C-Y, Sung J-M, et al. Cytomegalovirus disease in nonimmunocompromised, human immunodeficiency virus-negative adults with chronic kidney disease. J Microbiol Immunol Infect. 2014;47(4):345-9.

6. Shah R, Vaidya G, Kalakonda A, Manocha D, Rawlins S. Cytomegalovirus colitis mimicking rectal carcinoma in a young immunocompetent patient. ACG Case Rep J. 2015;2(3):165-7.

7. Huang R, Benmassaoud A, Mayrand S. Severe cytomegalovirus infection masquerading as recurrent ischemic colitis in a patient with end-stage renal disease. ACG Case Rep J. 2017;4:e126.

8. Yagel 0, Israel S, Muszkat M, Ackerman Z, Szalat A. Challenges in the diagnosis of cytomegalovirus colitis in a presumed immunocompetent patient: Interpretation of clinical condition, tissue, and serum cytomegalovirus polymerase chain reaction findings. Isr Med Assoc J IMAJ. 2018;20(3): 194-6.

9. Goodman AL, Murray CD, Watkins J, Griffiths PD, Webster DP. CMV in the gut: a critical review of CMV detection in the immunocompetent host with colitis. Eur J Clin Microbiol Infect Dis. 2015;34(1):13-8.

10. D'cruz RT, Lau CC-L, Thamboo TP. Severe ischemic cytomegalovirus proctocolitis with multiple perforation. Arch Virol. 2018;163(7):1927-31.

11. Hasegawa T, Aomatsu K, Nakamura M, Aomatsu N, Aomatsu K. Cytomegalovirus colitis followed by ischemic colitis in a non-immunocompromised adult: A case report. World J Gastroenterol. 2015;21(12): 3750-4.

12. Sager K, Alam S, Bond A, Chinnappan L, Probert CS. Review article: cytomegalovirus and inflammatory bowel disease. Aliment Pharmacol Ther. 2015;41(8):725-33.

13. Sudcharoen A, Chonmaitree P, Poonyam P, Trongwongsa T. Cytomegalovirus colitis in an end-stage renal disease patient presenting with lower gastrointestinal bleeding. J Med Assoc Thail Chotmaihet Thangphaet. 2016;99(Suppl. 8):S237-S243.

14. Brandt LJ, Feuerstadt P, Longstreth GF, Boley SJ. American College of Gastroenterology. ACG clinical guideline: epidemiology, risk factors, patterns of presentation, diagnosis, and management of colon ischemia $(\mathrm{Cl})$. Am J Gastroenterol. 2015;110(1):18-44. 